\title{
Study on the Social Amplification Risk Effect of Corporate Internet Reporting
}

\author{
Lingyan Ou \\ Department of Accounting, Fujian Jiangxia University, Fuzhou 350108, China \\ Financial and Accounting Research Center, Fujian Province Philosophy Social Science Research \\ Base, Fuzhou 350000, China
}

22087335@qq.com

Keywords: Corporate Internet Reporting, Risk, Social Amplification.

\begin{abstract}
The risk of corporate internet reporting has a significant impact on the decision-making behavior of the public, but how is the risk generated? Drawing on the social amplification framework of risk, the paper makes a theoretical description of the formation mechanism on corporate reporting risk. It is believed that the accumulation and formation of risk in a new environment can be divided into two stages: information amplification mechanism and information reaction mechanism of corporate reporting-based internet.
\end{abstract}

\section{Introduction}

Corporate internet reporting (CIR), mainly refers to the company's voluntary disclosure of information based on the internet, that is, the company actively and voluntarily disclose the financial and business information through the Internet platform. With the advent of self media era, and the wide use of twitter, micro-blog and WeChat, personal social platforms show strong communication power, then the information disclosure channels reported by internet are further expanding. The content and scope of disclosure are expanding, fragmented information guided and guided reading have always existed. Personal spontaneous communication has become a powerful force as the mainstream media, the information disclosure of listed companies enter the national reading and communication era. This is also the practical basis of this study.

Because of the economic consequences of accounting information, stakeholders, such as investors, creditors, and the public, constitute an important part of the mass media. In the current stage of the internet application of Web2.0, network information display based on the spread of powerful. The wide involvement of mass media and self media, cause the scope of disclosure has been extended from the initial statutory disclosure to a wide range of voluntary disclosure, especially through the establishment of the company website, micro-blog, WeChat or other network interaction open platform. Corporate reports are more closely linked to the mass media, and listed companies are also interested in the media. In fact, the rapid changes in the Internet and information technology, is quietly changing the traditional corporate financial reportings. In the Internet era, the risks of company reports are continuously derived and evolved, and new carriers, communicators and transmission channels need to be repositioned urgently. This is a new problem of accounting information disclosure under the Internet environment.

So, How is the risk formed? What changes have taken place in its elements, path of transmission and mode of transmission? Does mass media lead to social amplification of risks? What role does it play in the risk transmission of accounting information disclosure? These are important problems in the study of corporate reporting risk, and also the important research topics to solve.

\section{Theoretical Framework Interpretation}

The starting point of the paper is the new environment of the company reported risk. Cause the company reports involving the change of multi stakeholders and new disclosure environment transfer mode, risk factors which may affect the company report disclosure effect is increasing. New evolution 
of risk factors may be caused by new changes in people's behavior, values, self interpretation and additional information. Therefore, the article regards risk as the result of objective existence and subjective factor. As a potential threat or harm to the information users, the risk has a kind of realism. At the same time, it is given the label of social and cultural factors. Thus, risk may be biased by information users because of different interpretations.

So, the paper argues that the introduction of a comprehensive framework to enlarge the risk of social framework will be more comprehensive analysis on the Internet reporting risk, and this risk at least includes the following contents: one is the objective loss or injury of the participants of information transfer, two is risk consequences caused by the additional effect of culture and social factors in the process of information transfer.

\subsection{Framework for the Social Amplification of Risk.}

The social amplification of risk refers to the risk social experience and risk results under the joint action of information transmission process, economic system, cultural structure, individual and social group reflection, etc. The information transmission chain and the public response determine the nature and importance of risk. If out of the social category, the risk is likely to be reflected in the limited scope of the small impact, the consequences of the severity is also limited.

Obviously, the social amplification framework regards all kinds of factors in the social environment as information amplification stations. Producing and transmitting information through mass communication channels, at the same time, every information receiver is also involved in the process of information amplification, then taking measures to deal with risk according to social response. As shown in Figure 1 below.

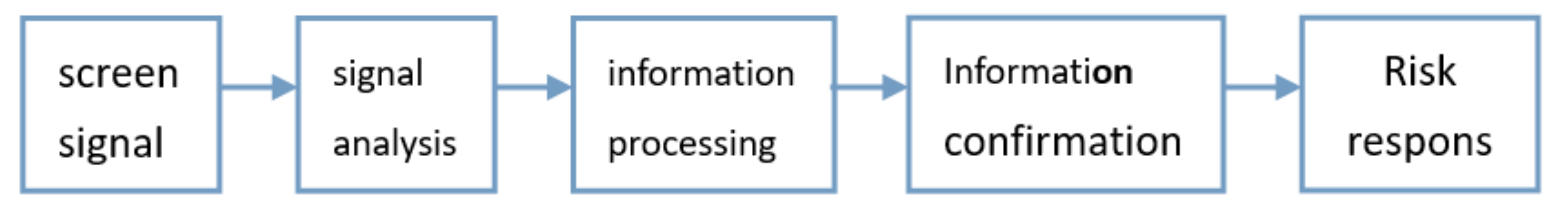

Figure 1. The process of information enlargement and response

\subsection{The Social Amplification Effect of Risk}

Researchers believe that the risk are discussed in the social amplification framework, is partly made up of direct threats of harm to people and the environment, and the other part is the decision (to people, companies, social organizations, and community values) associated with social cognition and social structure damage to the nature of the threat (Kasperson, 1992). Therefore, the framework of social amplification of risk (SARF) includes both ontological and epistemological categories of risk perception. Risk, as an objective threat or harm to people, has a kind of ontological realism. At the same time, risk is an element of the real world that is filtered by social and cultural factors, and has an epistemological instability. Obviously, the interpretation of risk is comprehensive.

The social magnification framework of risk discusses that, as a key part of the communication process, risk, risk events and their characteristics has been portrayed through all kinds of risk signal (image, signal and symbol). These risk signals in turn to enhance or weaken the cognition of risk and its controllability. Thus, this risk experience is not only a simulated experience of actual damage, but also the result of the group learning to interpret the risk process (Kasperson, 2000).

Based on the theory of communication, the social amplification framework for risk (SFAR), is used to analyze the ways in which various societies interpret, transfer and receive risk signals. At the same time, the signal may have changed in the society transfer process. This conversion can strengthen some of the information characteristics, such as increasing the information content, describing a variety of risk event symbolic images, leading to a variety of change of information receiver and social responses, and gradually spreading through various amplification station. The above link is the strengthen process of the risk.

Researchers believe that the social amplification will make the risk effect far greater than the original category, then may spread to other institutions originally independent, which is called the secondary effect of risk amplification or "ripple" effect. In addition, the risk framework of social amplification may lead to five risk amplification mechanisms: public perception and values, social group relations, signal value, stigmatization, and social trust. 


\section{The Formation Mechanism of the CIR Risk Ripple Effect}

From a whole point of view, the formation of corporate reporting risk is divided into two major links, including the internal risk generation and external risk social amplification. The social amplification process can also be divided into two stages: the first stage is the information mechanism of social amplification, the second stage is the social amplification reaction mechanism, as shown in Figure 2 below. The paper believes that external social amplification is the decisive link of risk formation effect. If there is a lack of external information transmission, then the risk factors confined within the company will not form a complete chain, and the secondary effect of the company's reporting risk cannot be formed.

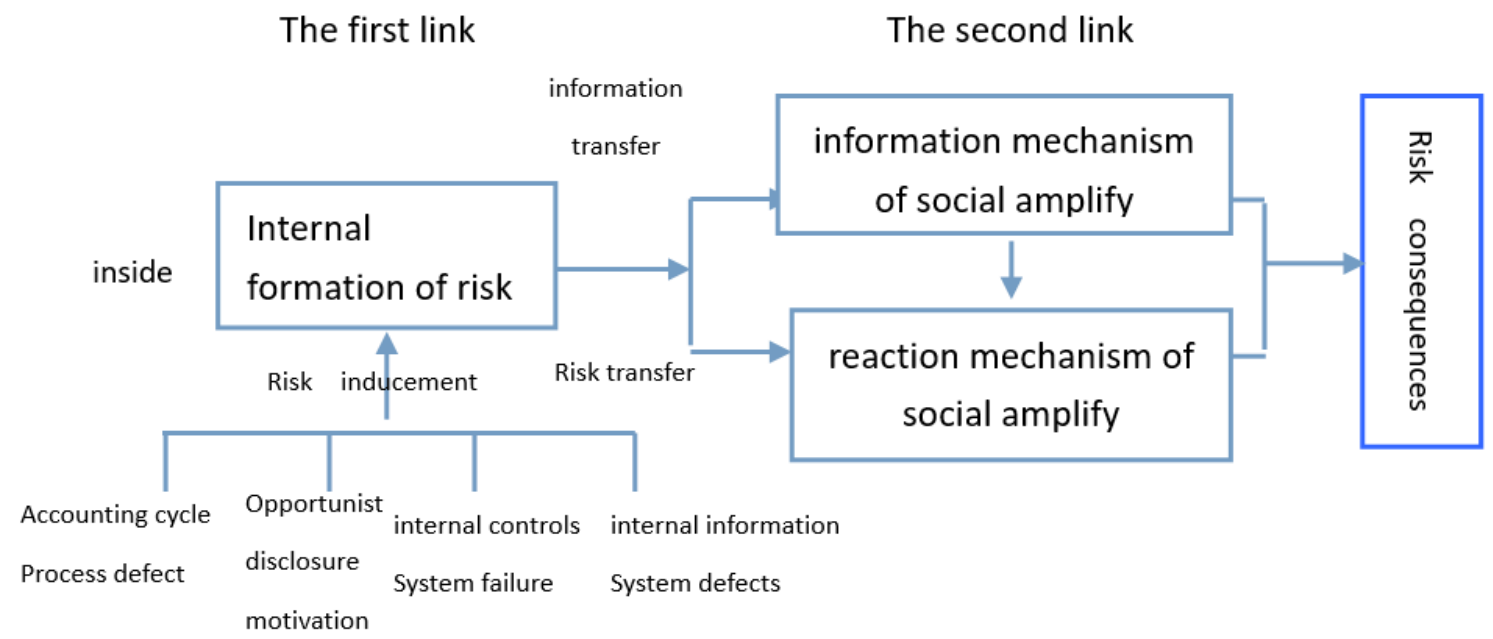

The accumulation of risk flow -- threshold value -- breakout

Figure 2. The formation mechanism of corporate internet reporting risk

\subsection{Internal Generation Mechanism of CIR Risk.}

The source of corporate reporting risks comes from the disclosure of information - the inside of the enterprise. In the process of accounting cycle (recognition, measurement, recording and reporting), there are various hidden dangers that may lead to information risk. The most likely factors are: (1) defects in the accounting cycle process; (2) opportunist disclosure motivation; (3) internal control system failure; (4) internal information system defects.

\subsection{Social Amplification Mechanism of CIR Risk.}

\subsubsection{Changes in Value Added Signals}

Previous studies have shown that the added value signal in information can arouse the attention of the information receiver, while it is the key role in information decoding (Hovland, 1984). In the process of information dissemination, the value signals attached to itself will arouse the strong concern of the recipients. We believe that the CIR risk is amplified at least two times, namely, the stage of information reception and the information re-encoding stage. These two stages may lead to the change of the added value of the original company's report information.

\subsubsection{Two Transmission Magnification of the Corporate Reporting}

This article boldly suggests that the social amplification and dynamic transmission of the company's reporting process on the Internet eventually lead to the dominant result of the risk, that is, the secondary effect. In particular, with the development of mass media, the public has completed two possible transmissions or releases with the help of media channels. Each transmission makes itself the second source of transmission. In this process, the interpretation of the original company's report information makes the risk signal magnified, while the theory of six-dimensional space has greatly deepened this disordered amplification and dissemination. "six degrees space theory" also called "six-degree segmentation theory" or "small world theory", that is, any two people in the world can be linked through the chain of friends within six layers. 


\subsubsection{The Flow of Information in the Mass Media Drives the Risk Magnification}

Risk is closely related to personal and social experience. To a certain extent, people's experience of risk has become the root cause of the risk society's enlargement. Since it is impossible for people to experience all the mentioned risks directly, the knowledge of risk is largely derived from public media, as well as public channels. Therefore, the information flow provided by the media constitutes the important factor in the risk perception of the public and constitutes one of the main driving forces for the risk amplify.

Among them, the most important factors are: (1) the number of information reported by the company (the original media and the public Reprint); (2) the degree of information controversy; (3) the "dramatic" degree of the company's report information, once false or exaggerated information sources have entered the media, even very small events can cause a huge rebound because of the "ripples" effect.

\subsubsection{Expansion of Information Disclosure Channels}

In the process of company report disclosure, information channel is an important factor for risk formation and avoidance. Generally speaking, mass media and informal interpersonal communication networks are the main channels of risk communication.

\subsection{The Social Amplification Reaction Mechanism of CIR Risk.}

The amplification mechanism of risk society is the interpretation and response of information flow. In this process, risk information is interpreted, identified and added to the value of self-cognition or social cognition. This process is obviously subject to the background of society, culture and system. The mechanisms of action may be presented in four ways as below:

\subsubsection{The Risk Response of the Public}

A large number of cases show that some existing simplified processes or mechanisms may be used by individuals to deal with risk assessment, control and consequent social reactions. This process is sometimes accompanied by the distortion and error of personal preferences (Kahneman et al., 1982). The company report has economic consequences; therefore, individual and group attention will cause different reactions to their interpretation. Generally speaking, people will analyze from previous cases, interpret the possible risk factors that may be hidden in the information, and then choose the possible risk consequences.

\subsubsection{The Game Angle of Social Groups}

In the field of internet corporate reporting risk, various social groups, such as investors, creditors, regulators, and the public, have formed a game of all kinds of risk interpretation and control, with different opinions on the possible risk consequences. They coordinate risk tolerance and risk management measures, and the coordinated consequences will greatly affect the social response.

\subsubsection{The Mutation of Risk Signal Value}

As a carrier of information transmission, the risk signals contained in the company report and its transmission process are different. To some extent, the deep impact of risk and its social effects can be predicted by the signal of risk information. Obviously, high risk signals are more likely to be captured by the external environment, and people are more willing to discuss the possible consequences of high risk signals. Therefore, in the process of company reporting transmission, the mutation of the signal value is an important factor needs attention. It often represents the breakthrough of the internal risk critical threshold in the corporate reporting and can be continuously fermented and magnified with the help of the social network.

\subsection{4. "Stigma" Consequences of Information}

For the capital market, the stigma effect is very common. Because of the economic consequences of accounting information, the information release is casual and unregulated under the environment of the media. The supervision of information release is loose, and many unconfirmed or deliberately misinterpreted information will spread more easily and lead to direct economic secondary effects. There are also some cases of market fluctuation caused by media reports and over reading in the capital market. 
It should be said that such stigmatization is very common, especially when a certain information is concerned by a high level of social concern, then the risk associated with the interest may be magnified, and the social response may also lead to positive feedback on the risk itself.

\subsubsection{Whirlpool of Public Opinion}

Because of the rapid development of modern mass media and various kinds of self-media, the speed and scope of information spread are in a disorderly growth of geometric multiplier, which can easily lead to uncontrollable public opinion. A large number of hearsay is likely to cause "stigma" situation. No matter for enterprises or investors, it will cause serious risk harm. In the disclose of corporate internet reporting, the role of media whirlpool is subtler. In particular, self-media voice makes a number of different interpretations of the company's reports, including the "noise" that deviates from the facts and rationality, which makes the public's fear of risk likely to be reflected in public opinion. In fact, these risk consequences are likely to be triggered by a risk factor that looks less threatening. It is clear that mass media have the ability to create a "whirlpool of public opinion", and a large number of public opinion storms can be combined into one impulse, which may lead to unpredictable and estimated results.

\section{Conclusion}

The paper is starting with the concept of corporate reporting risk, believes that the risk problem is a real existence which cannot be ignored, especially the important invisible control of media force in promoting public opinion. There are some practical problems that need to be solved urgently, such as the release of false information, the untimely clarification of adverse reports, and the impression of "stigmatization" in the public heart, and so on.

The social amplification framework of risk is used to describe the formation mechanism of corporate reporting risk. It is considered that the accumulation and formation of corporate reporting risks under the new environment are two stages: the first is the information amplification mechanism of the company report, and the other is the information reaction mechanism of the company's report. The formulation of risk aims at providing an overall framework for subsequent research on quantitative analysis of risk.

\section{Acknowledgments}

This work is financially supported by Education and scientific research project of young and middle-aged teachers in Fujian Province Research on the social magnification effect and control mechanism of company online reporting risk (No. JAS170553).

\section{References}

[1]. Joanna Dyczkowska. Assessment of Quality of Internet Financial Disclosures Using a Scoring System. A Case of Polish Stock Issuers. Accounting and Management Information Systems, Vol. 13(2014), p.50-81.

[2]. WJ Burns, P Slovic, RE Kasperson et.al. Incorporating structural models into research on social amplification of risk: Implications for theory construction and decision making. Risk Analysis, Vol. 13(2010) No.6, p.611-623.

[3]. PL Poon, YT Yu. Degree of Internet corporate reporting: a research framework. Information Research, Vol. 17(2012) No.1, p. 253-270.

[4]. LY Ou. The risk of corporate information disclosure-based internet and three-dimensional regulation mode. Proceedings of the 28th Chinese Control and Decision Conference, CCDC2016. p.4491-4496.

[5]. David Robb, Fiona H. Rohde and Peter Green. Standard Business Reporting in Australia: Efficiency, Effectiveness, or Both. Accounting \& Finance, Vol. 56(2016) No.2, p. 509-544. 
[6]. E Oluwagbemiga. The use of voluntary disclosure in determining the quality of financial statements: Evidence from the Nigeria listed companies. Serbian Journal of Management, Vol. 9(2014) No.2, p. 263-280. 